\title{
Application of Quilting Art in Interior Furnishing Artistic Design
}

\author{
Ji-Chun $\mathrm{LI}^{1}$, Yuan YANG ${ }^{1, a,{ }^{*}}$ \\ ${ }^{1}$ Hubei University of Technology, China \\ a591452410@qq.com \\ ${ }^{*}$ Corresponding author
}

Keywords: Quilting Arts, Interior furnishing, Application.

\begin{abstract}
Fabric products, with its characteristics like rich colors, diversified patterns and function of softening rigid space, have attracted great attention from designers. Meanwhile, quilting products, which is one variety of fabric products with a strong flavor of artistic, will result in favorable effects when applied in interior furnishing design. This paper, through sorting out the development history of quilting art and exploring for the beauty of quilting art, has focused on the application of quilting art in interior furnishing design and concluded some principles of applying the art in interior furnishing design for discussion with a view to promoting the development of interior design.

Quilting Arts has generated new artistic charm with the fun of "quilting", abundant color compositions, modern artistic forms that have been decomposed and restructured and the abundant texture effects resulted from its combination with modern advanced sewing technologies. With the rise of industrialization of quilting DIY, quilting art is finding its way back to the scene and the ancient folk handmade quilting has become synonym for fashion art and leisure culture for urban people in the 21st century. The application of quilting art in interior furnishing design and recombination of artistic quality and practicability of quilting will not only result in desirable decorative effects in interior space but also play a critical role in the inheritance, protection and innovation of traditional Chinese culture.
\end{abstract}

\section{Origin and development of quilting art}

Quilting, a craft for piecing small fabrics of various shapes according to certain rules, was originated from folk handmade crafts in ancient Egypt and northern area of China. In Egypt, the earliest quilting form can be traced back to various quilting clothes on statues of pharaohs of Egypt. In China, quilting can be found in pull-over garments and skirts of the Spring and Autumn period that have been founded in No.1 cemetery of Zhagunluke ancient tomb group in Qiemo county of Sinkiang. Up until now, more than 2, 000 years have passed. As an important member of Chinese needlework culture, quilting is widespread in China. In ancient agricultural society where supplies were in shortage and medical treatment and health level was lagging behind, there was a high probability for children, especially infants, to die. In order to bless children, common people began to sew clothes and quilts made of patches for newly-born infants. Moreover, the patches were primarily small fabrics or old clothes given by relatives or neighbors. When the patches were sewn into clothes and quilts, they were placed with the implication of blessing from one hundred families. Consequently the good connotation of patches of driving away disasters and evil things and praying for bless and auspiciousness was playing an important role in China's folk culture and there were clothes, ornaments and tools for children made of patches everywhere in the folk. In modern times especially since the reform and opening up, however, the custom of patches has been disappearing in the folk with the growing of richness of materials and popularity of industrial products. As a result of that, some handicrafts are in a dilemma of getting lost.

In European and American countries where quilting art had a similar origin, women who participated in the Women's Lib in 1970 made quilting products for public welfare activities and gave them to hospitals. It is safe to say that they have saving quilting art through activities for relieving the poor. After a long period of development, European and American countries have developed a 
distinct quilting style which is changing from pragmatism-oriented to omamentalism-oriented with the improving of living standard. Later, quilting art began to prevail in Japan and many Japanese women regarded quilting as a hobby. In the process of development, Japanese quilting has taken the way of industrialization.

In China, the development of quilting art has taken shape with the showing of a promising market prospect. However, relevant books in China are focusing on quilting production and there is a shortage of relevant literatures with regard to application of patch-based artworks. This paper, through a great deal of examples and analyses, is aiming at providing certain reference for applying patch-based artworks in interior furnishing.

\section{Aesthetic value of quilting art and interior furnishing design}

The beauty of quilting art lies in its bright colors and textural effect as well as its form after being decomposed and restructured and its thought-provoking implication. Nowadays, the flourishing of interior decoration art and urban people are longing to return to origin, quilting art has gradually come to the scene of the public in its unique aesthetic form. In common sense, interior furnishing art primarily refers to the furnishing and combination of artworks and ornaments. With the improvement of people's living standard and rapid development of interior decoration industry for families and public places, various interior design styles and schools are competing with each other for beauty of looks. On the other hand, the unique aesthetic form of quilting art is catering for the art era when various styles are competing with each other. Moreover, it has enhanced some of the styles while indicating personalized colors of interior decoration design.

\section{Aesthetic value of materials of colors and texture and design of texture school}

Since the aesthetic design of texture school is to increase artistic strength of interior design through emphasizing textural effect of materials, the school is characterized by full displaying of textural effect and features of materials in interior design and utilization of modern high-tech processing art for creating and fully displaying new textural effects of the materials. The materials are either powerful or highly precise and delicate, either dense and complex or plain and simple, either luxurious and graceful or artless and vivid. Therefore, displaying of textures of these materials is likely to provoke affection and association among people and guide people to intervene in certain atmosphere and experience the artistic conception. In summary, quilting art is a means for presenting design ideas of texture school.

Firstly, materials used in patches include cotton, linen, silk, fur and suede. Among them, the common cotton, linen and silk are woven with cotton, jute and natural silk respectively, so they are featured with natural colors and aesthetic perception. Meanwhile, materials of cotton and linen are warm, comfortable and unsophisticated; the silk is bright in color, noble and romantic, displaying a riot of color and rich emotion after being dyed and print. Furthermore, there are many styles for fabrics, such as the blue print fabric that is capable of highlighting traditional Chinese art, the yarn-dyed fabric that feels rustic, the reminiscent country style that contains pictures of stars, flags, hearts, farmhouses, corps, animals and words, and the romantic pastoral style based on pictures of colorful flowers. Hence, various fabrics have been pleasant to eyes before being pieced. After careful composition and matching of colors, the different textures, colors and styles will generate unique charm when coming together.

Secondly, a range of different "piecing" skills have been generated in the process of doing quilting due to limited materials and a pursuit for superb artistry, such as patching, darn, stitch, piece, paste, edge, pile and sew. Moreover, a great deal of typical hand stitches that remain popular nowadays have been created, such as plain stitch, hidden stitch, back stitch, a thousand-bird stitch, large running stitch and roll stitch. From artistic perspective, different stitches have different decorative effects. In addition, selection of stitches should be made according to characteristics of elements of shapes of quilting products, such as point, line, surface, color and quality. The reason is that hidden stitches and 
revealed stitches, along with dense stitches and loose stitches, will result in totally different textural visual effects. Meanwhile, the invention of modern automatic sewing machine has not only improved the efficiency of quilting but also increased the unique forms of artistic presentation, including "splicing of small pieces" (fig. 1), "appliqué stitch"(fig. 2) and "top stitch" (fig. 3). To sum up, quilting art is to certain extent representing the design of texture school. Secondly, a range of different "piecing" skills have been generated in the process of doing quilting due to limited materials and a pursuit for superb artistry, such as patching, darn, stitch, piece, paste, edge, pile and sew. Moreover, a great deal of typical hand stitches that remain popular nowadays have been created, such as plain stitch, hidden stitch, back stitch, a thousand-bird stitch, large running stitch and roll stitch. From artistic perspective, different stitches have different decorative effects. In addition, selection of stitches should be made according to characteristics of elements of shapes of quilting products, such as point, line, surface, color and quality. The reason is that hidden stitches and revealed stitches, along with dense stitches and loose stitches, will result in totally different textural visual effects. Meanwhile, the invention of modern automatic sewing machine has not only improved the efficiency of quilting but also increased the unique forms of artistic presentation, including "splicing of small pieces" (fig. 1), "appliqué stitch"(fig. 2) and "top stitch" (fig. 3). To sum up, quilting art is to certain extent representing the design of texture school.

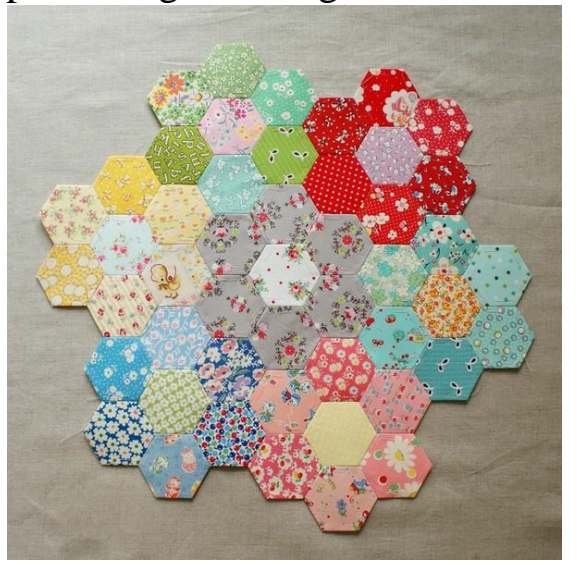

Fig 1 splicing of small pieces

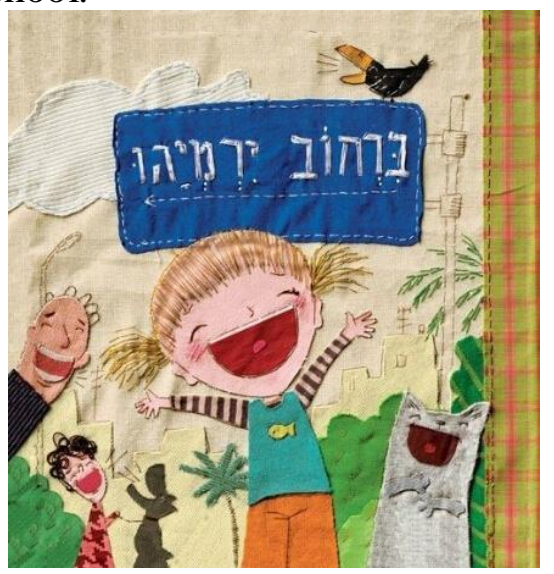

Fig 2 appliqué stitch

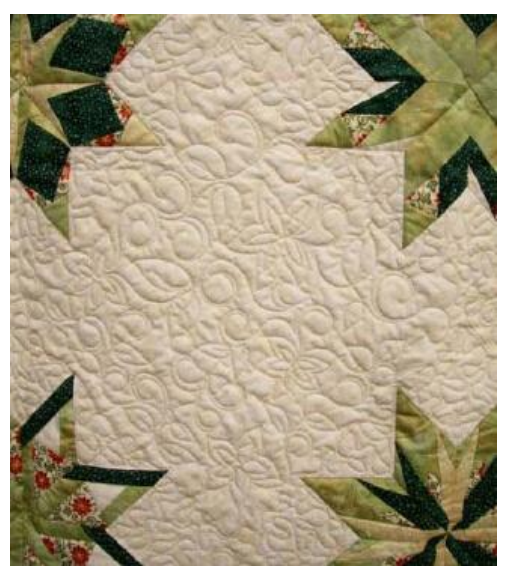

Fig 3 top stitch

\section{Formal beauty of decomposition and reconstruction and postmodernism design}

The "post" means revolt, that is, revolt against modern art. According to postmodernists, design is not only for solving functional problems, but that emotional factors of human being should also be taken into account. In other words, they advocate for emotional design and oppose simple modeling; moreover, they are particular about context, pursuing for humanity, worshipping using of metaphors, symbolism and a great deal of decorative colors and calling for diversified designs. On the other hand, quilting art is catering for these features of postmodernism design.

Firstly, postmodernism design regards design as for serving people with regard to design philosophy and an amiable and free dialogue between human being and products. As a handicraft with strong national color, quilting art is different from mass production of industrialization due to its fine workmanship and meticulosity. Therefore, it is more likely to generate a sense of identify and sense of belonging among people. Moreover, quilting products can also be completed by a host through DIY and such products can fully present interests and life quality of the host while narrowing the distance between human being and interior decoration design. Secondly, postmodernism is used to using deconstructivism which is one kind of structuralism as well as an anti-traditional structuralism that has been reshaped with new perspectives and thinking. Essence of quilting lies in the artistic form of "quilting". Since quilting is a restructuring of tailored fabrics according to a new rule of formal beauty, it is consistent with structuralism with regard to design pattern. Rules of formal beauty that are often applied in quilting art design include repetition, gradient, comparison, balance, rhythm, emission and texture. Furthermore, basic shapes that are constituting quilting products 
include square, triangle, rhombus and other geometrical shapes and natural shapes like shapes of flowers, animals and teapots. These shapes, when combined according to rules of formal beauty, will constitute a wide range of quilting artworks (fig. 4 and fig. 5).

\section{The design philosophy of postmodernism is influencing traditional quilting art}

Traditiona quilting is particular about the harmonious beauty between colors and their implications. Therefore, in aesthetic design of postmodernism, harmonious colors have evolved into aesthetic fatigue in the new era and anti-traditional colors for highlighting individualities have been used in the artistic design of quilting, such as Elle decorative quilting furniture (Fig 6).

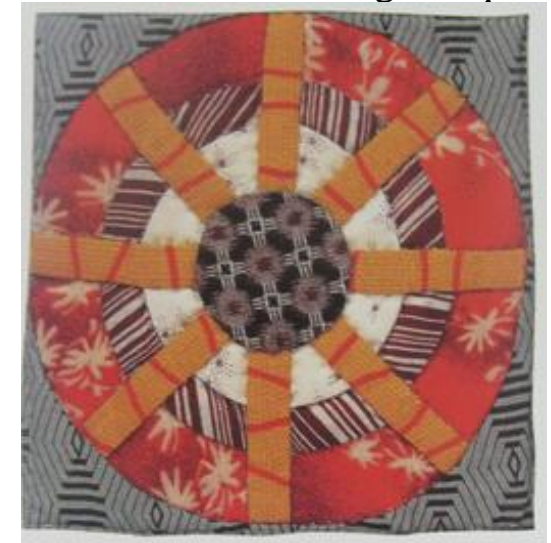

Fig 4 circles • curves
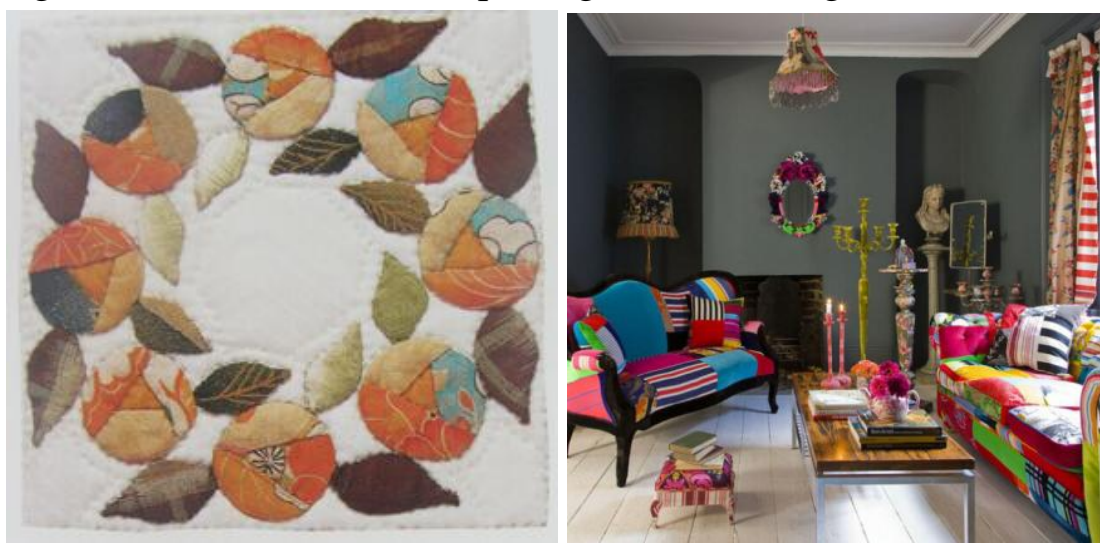

Fig 5 Garland

Fig 6 Elle decorative quilting furniture

\section{Application of quilting art in interior furnishing artistic design}

In interior space furnishing design, pay attention to relations between models and colors and between ratio scales and space when choosing quilting artworks; meanwhile, pay attention to the relationship between connotation of a work and interior style. In interior decoration design, the proportion of quilting products should not be too high cause it may result in visual crowdedness and a failure to achieve the effect of highlighting important points which, when being overlooked, may ruin the harmonious interior atmosphere and environment.

\section{Application of quilting products in interior plane display}

Since most plane artistic decorates are hung on walls or spread out on ground or platforms, they belong to interior space while having relatively independent artistic value. They are not only decorating the space but also balancing relationship among dimensions of interior space. In general, quilting products are in forms of wall hangings, tablecloths, bedspreads, bedclothes, cushions and coasters.

When Since most plane artistic decorates are hung on walls or spread out on ground or platforms, they belong to interior space while having relatively independent artistic value. They are not only decorating the space but also balancing relationship among dimensions of interior space. In general, quilting products are in forms of wall hangings, tablecloths, bedspreads, bedclothes, cushions and coasters.

Firstly, matching design of colors, patterns, materials and textures. The selection of colors and patterns of quilting should be determined according to the style of interior space. Moreover, colors of furniture should be matched through selection similar colors, contrasting colors and similar patterns, for example, cushions should be designed to match tablecloths and table mats in the space of a dining room. Moreover, taken characteristics of the dinning table and chairs into account and fully absorb the environmental color to achieve an unified visual effect. As shown in fig.7: the quilting cushions in the dinning room give soft touch for people; in addition, warm colors are selected for matching and color of every piece of fabric is similar to the surrounding environmental color, corresponding to colors of the table mats; finally, patterns of the cushions are primarily based on small pieces with 
floral print and grid print, corresponding to patterns of the dinner plates to achieve a harmonious and unified visual effect. As with space of bedrooms, quilting art products are needed for improving a feeling of warmth and degree of visual fitness in the interior space. The fig. 8 presents space design of a bedroom with a simple style. In the bedroom, the big quilting artistic wall tapestry is functioning as background wall of the bed while playing a role of dividing the space. Besides that, most colors of the tapestry are similar to colors of the ochre walls, redwood furniture and black photo frame. Visually, the tapestry has been integrated into the environment perfectly, contrasting to the white bed clothes. As with the colors of the bed clothes, pure white color is not applied thoroughly; instead, quilting design corresponding to the tapestry is applied for creating a simple and cozy visual effect for the space.

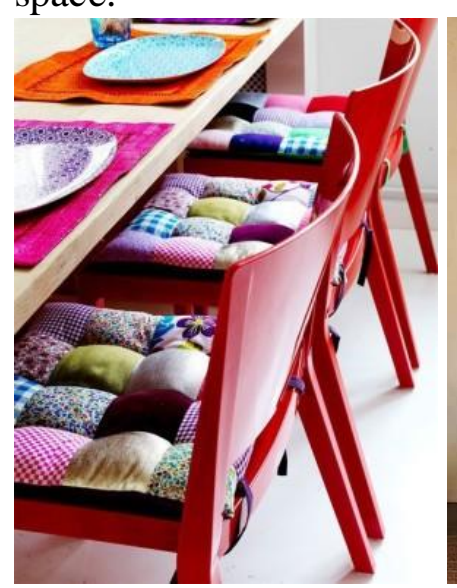

Fig 7 One corner of the dinning room

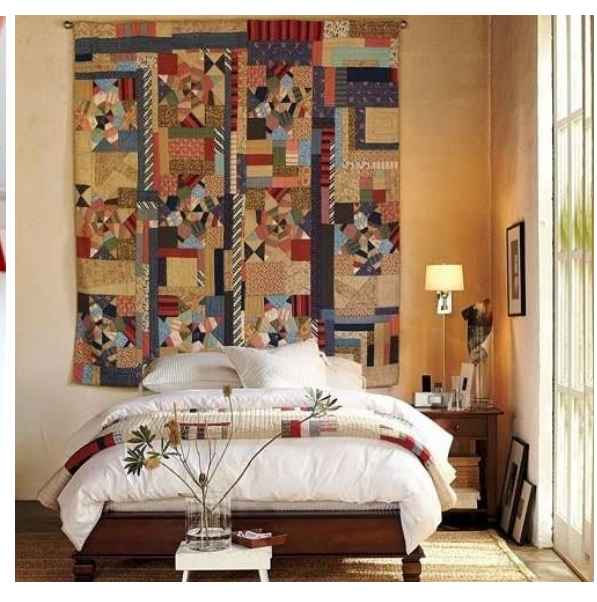

Fig 8 and Fig 9 Design of bedroom space based on quilting art

Secondly, matching design of forms and styles. In the area of interior space design, different colors, patterns, creation ideas and design methods will result in different colors and styles for interior space. Therefore, we can design quilting artworks according to different themes and styles of interior space since an appropriate quilting artwork can enhance style of interior design. The fig. 9 presents design of a bedroom for children. In the bedroom, colors like pink green, pin blue and light yellow are used for creating breath of spring. With respect to selection of materials of quilting, common materials are cotton and linen for providing natural protection for children. Finally, patterns of the quilting include nightingale, little bird, big tree and so on for creating an innocent and romantic space for children. In summary, the overall design looks natural and reasonable.

Thirdly, overall design of furnishing and positioning. When furnishing quilting artworks in interior space, give consideration to overall design and use symmetric layout with sense of stability in solemn scenarios; in cozy, free and lively residential space, however, flowing asymmetric layout is often used; in occasions with single function and small areas, centralized style is often used; in occasions with multiple functions and large areas, distributed style is common.

\section{Application of quilting artworks in interior three-dimensional furnishing}

In general, three-dimensional artistic furnishing is for displaying works with three-dimensional space while basing on use functions. In spite of that, it is playing an important role for beautifying interior environment and creating certain atmosphere. Primary forms of quilting art in interior three-dimensional furnishing include furniture, pillow, quilting artistic puppet and so on.

Playing an important role in presenting environmental functions and creating artistic atmosphere in interior space, furniture can tactfully organize and divide space. Moreover, its shapes and colors can directly present the design style. Furthermore, quilting art is often integrated into design of furniture, such as the Victoria-style furniture shown in fig. 10. The furniture is graceful in line, like an elegant lady; meanwhile, the quilting of contrasting colors and complicated cloth furnishing have fully presented the bourgeois sentiments of people in Victorian era.

As accessories for chairs, sofas and beds, pillows can not only make up for deficiencies of some furniture in use function and increase the degree of comfort for the furniture but also adorn interior 
space. Moreover, exquisite pillows put in interior space will add taste to life and double aesthetic perception. As shown in fig. 11, the quilting pillows put the finishing touch to the interior space. Meanwhile, pillows are things that are easiest to be made through DIY. They are composed of lining, inner materials and filler; common fabrics for quilting pillows are blended fabrics, cotton cloth, velvet, cotton poplin, ramie cotton fabric and so on. People can choose materials according to personal preferences and create personalized quality life through DIY. Other quilting artworks that are easy to make through DIY include quilting artistic puppets, storage boxes, coasters, etc.

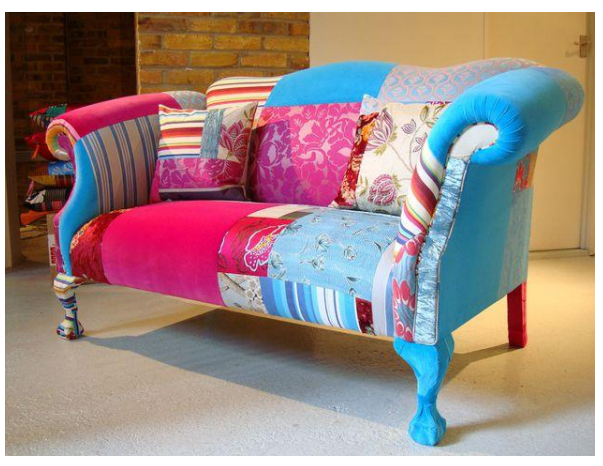

Fig 10 quilting furniture of Victorian style Fig 11 quilting pillow

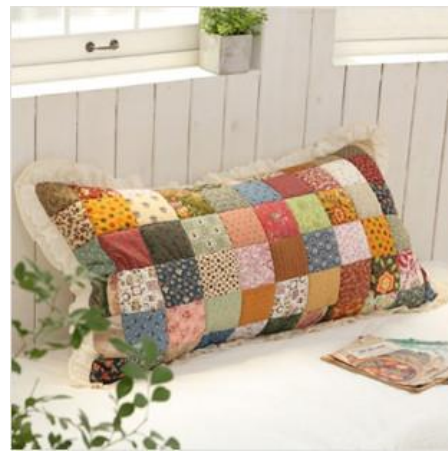

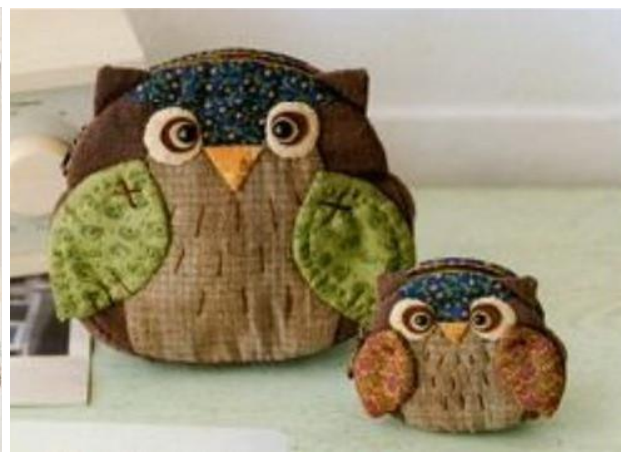

Fig 12 DIY handmade quilts

Under the attack of machine-sewn quilting, handmade quilting used to disappear for a period of time. With the speeding up of pace of life, however, people are eager to pursuing for restoring the soul. Consequently, a trend of DIY has been evoked across China and DIY quilting (fig. 12) has won a place for itself in the busy urban life with its distinct artistic expressive force and practical functions. It has become a way of leisure for people to imbue affection. Through quilting, people can directly express their colorful inner world while cultivating the mind. Besides that, completed quilting works can also be used for furnishing living space by adding unique personalized colors to the space, which is also the social background for the popularity of quilting art.

\section{Summary}

As an ancient and emerging art, quilting has long history. Currently, it is being widely used in fields of cloths, home textiles, and artworks. In interior decoration design, quilting products can be used in some places for adorning the space and creating certain atmosphere. Moreover, they can be used in a large scale for emphasizing personalized colors of space. Furthermore, they can be made by hosts through DIY for creating interior space filled with delight of life. In conclusion, interior space adorned with quilting artworks is free and cozy and filled with personalized colors. Besides that, it is unsophisticated while exquisite and filled with artistic flavor; furthermore, such space is fashionable, vivid and capable of presenting passion of its host towards life. Finally, introduction of quilting art into the field of interior furnishing design is a trend of personalized interior space design.

\section{References}

[1]M. Jin Guo Sheng,IArtistic Design of Interior Furnishing , Hangzhou :Zhejiang,People's Fine Arts Publishing House, 2011.7 (in Chinese).

[2]J. Su Miao, Primary Exploration on Application of Textile Products in Modern Interior Space Environment, Silk (in Chinese).

[3] J. Zou Xi, Exploration for Culture of Quilting art and Aesthetic Features , National Culture, (2012)6-0103-06.

[4] J. Soup,Ponders Over the Quilting Art of America, Zhuangshi,216th, 2011.04 (in Chinese). 